
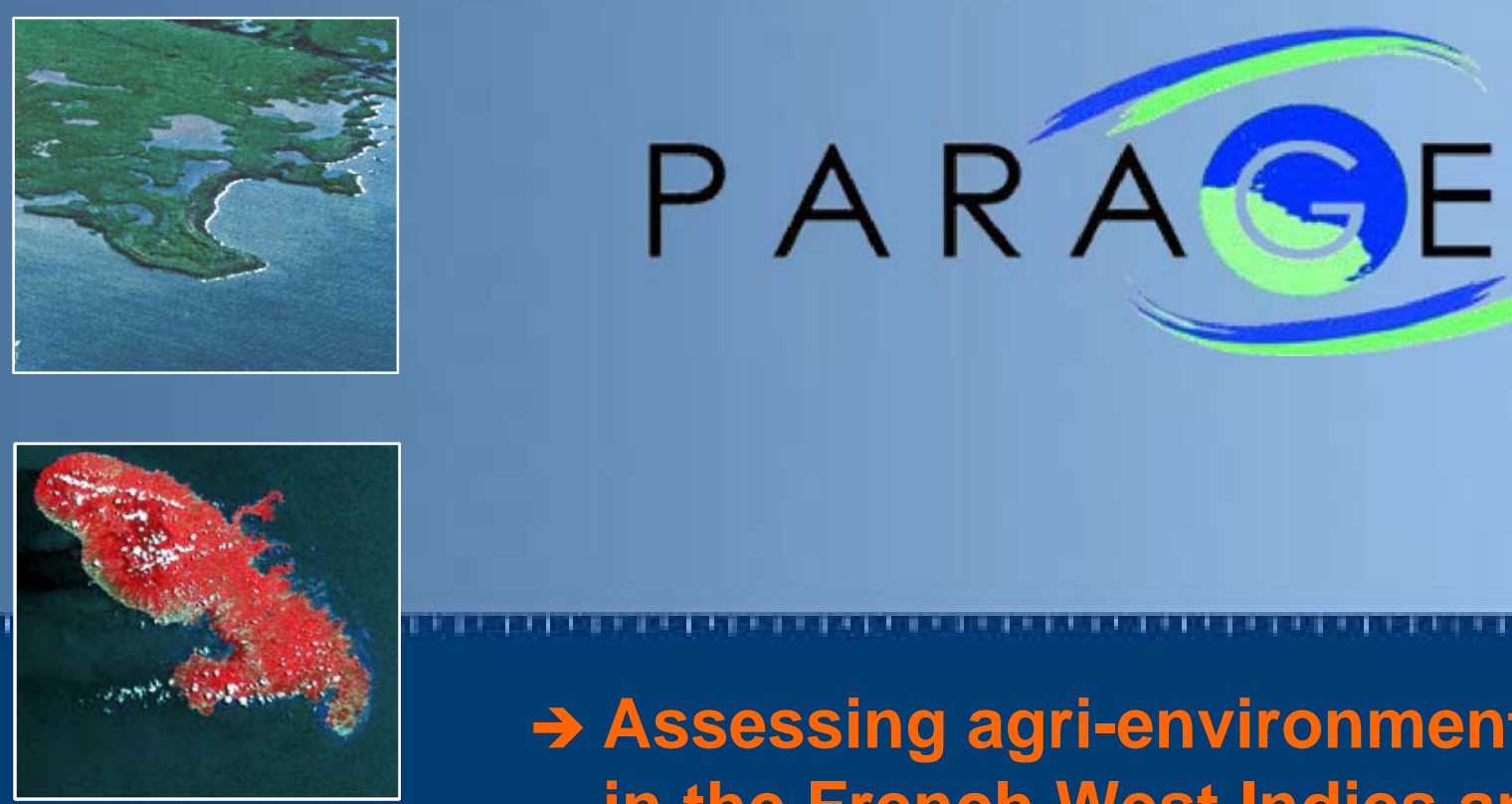

$\Rightarrow$ Assessing agri-environmental impacts in the French West Indies and

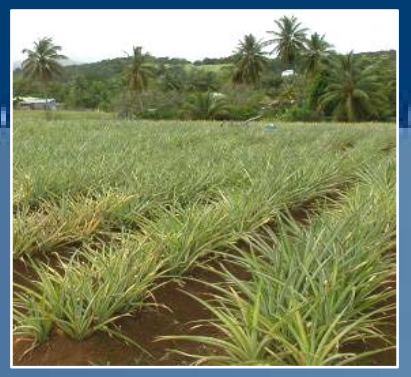
\title{
French Guiana
}

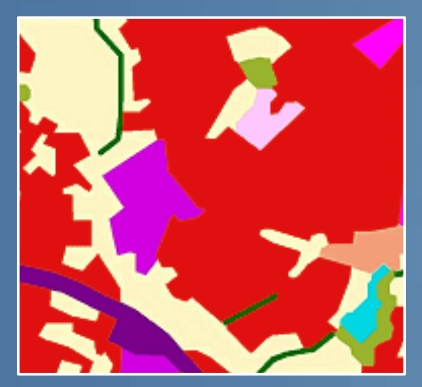

F. Bain II ariin ${ }^{1}$,

G. Lai né2,

B. Ari aux ${ }^{3}$,

D. Desprès ${ }^{3}$, J. M Fotsi ng ${ }^{4}$, K. Ose ${ }^{4}$, G. Gonzal es ${ }^{1}$

SIG

${ }^{1}$ Spot I mage, ${ }^{2} \mathrm{Cl} \mathrm{RAD},{ }^{3} \mathrm{SI}$ Gbea, ${ }^{4} \mathrm{RD}$ $50 \% \mathrm{f}$ unded by French $\mathrm{M}$ ni stry of Agri cul ture

$\rightarrow$ 28th EARSeL Symposium and Workshops, 2-7 June 2008, Istanbul, Turkey 
$\rightarrow$ PARAGE :

Assessing Agri-Environmental impacts in the French West Indies and French Guiana :

A space-based tool for assessing environmental impacts

- Innovative study on the evaluation of the impact of farming practices on natural environment

- Project co-funded by the French ministry of agriculture and fisheries

- 2 years project : March 20, 2006 - March 27, 2008 
$\Rightarrow$ Project objectives

- To evaluate the contribution of remote sensing technology for the agrienvironment diagnosis in the French West Indies and French Guiana

$\square$ Support for the creation and updating of agricultural and natural land cover maps

$\square$ Support for the set up of regulations regarding natural resources and territorial management

$\square$ Risk assessment and monitoring

- To strengthen cooperation between users

$\square$ Sharing of geographic information

- To demonstrate the contribution of the SPOT satellite image receiving station set up in French Guiana in Feb 2006

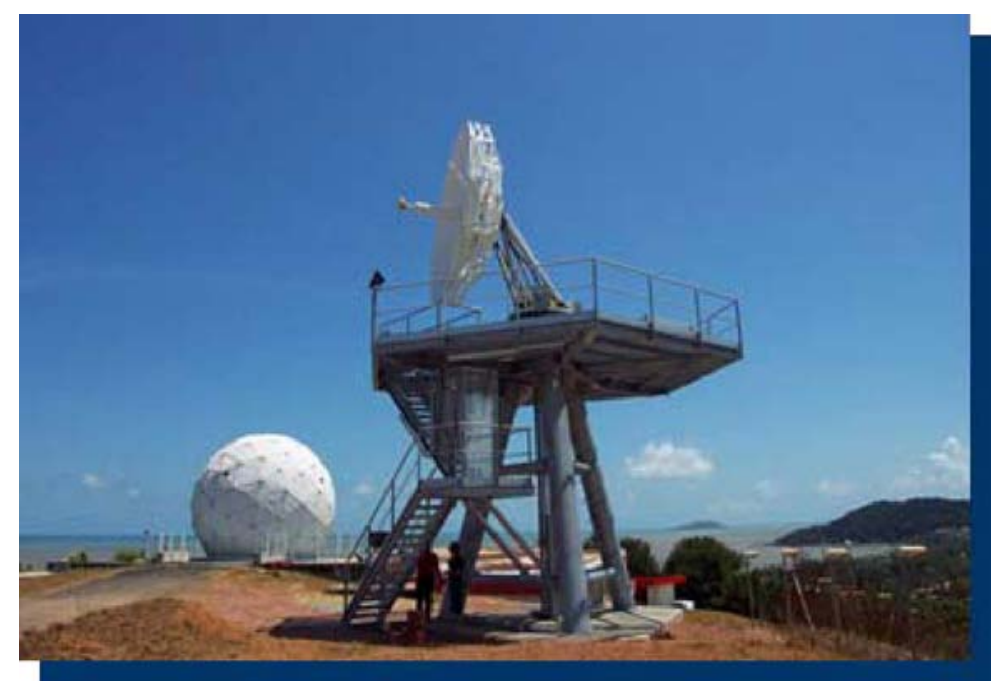




\section{$\rightarrow 3$ regions with different issue}

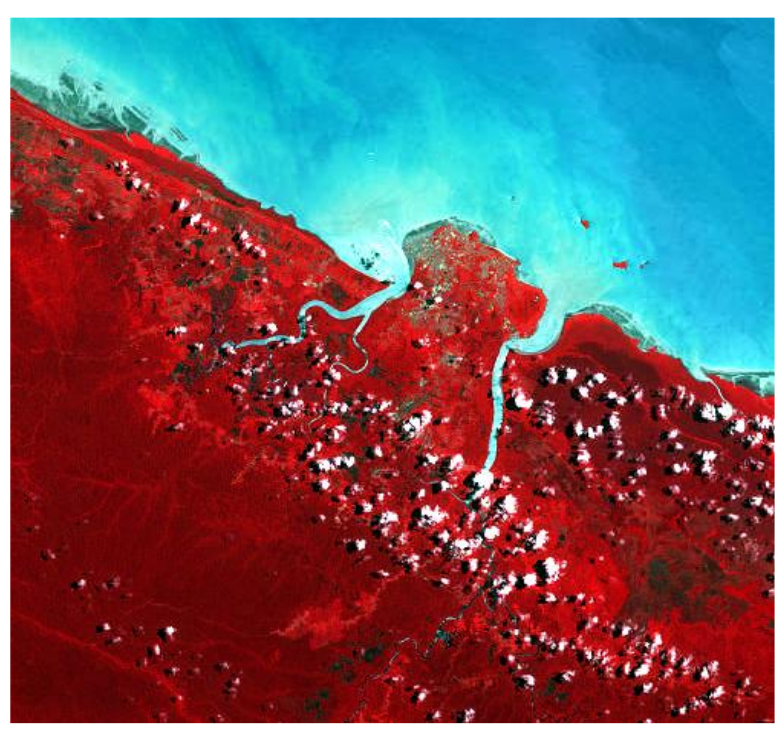

\section{French Guiana}

Deforestation monitoring for slash-and-burn agriculture

\section{Guadeloupe}

Evaluation of agricultural pressure over sensitive or protected environments

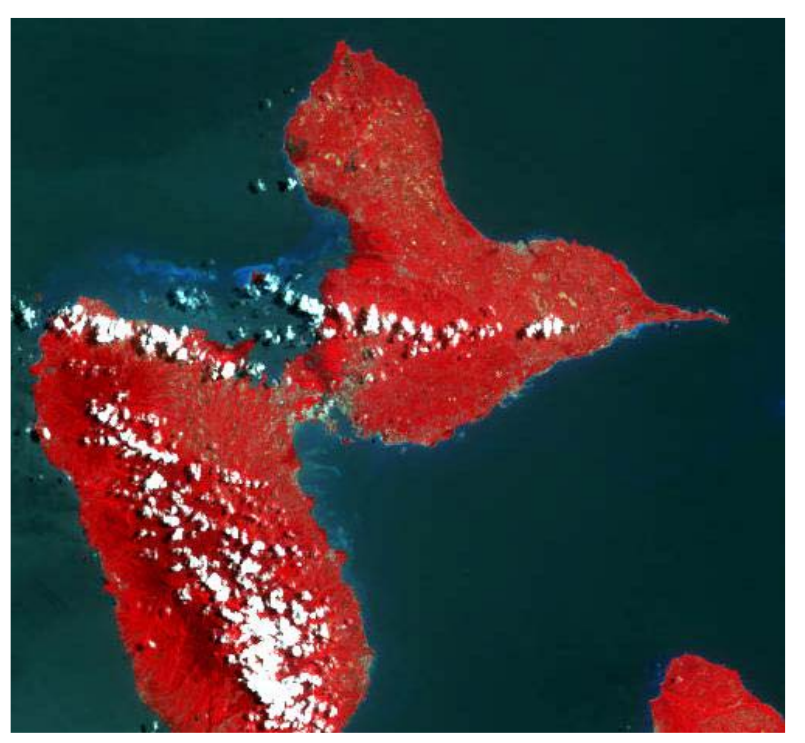

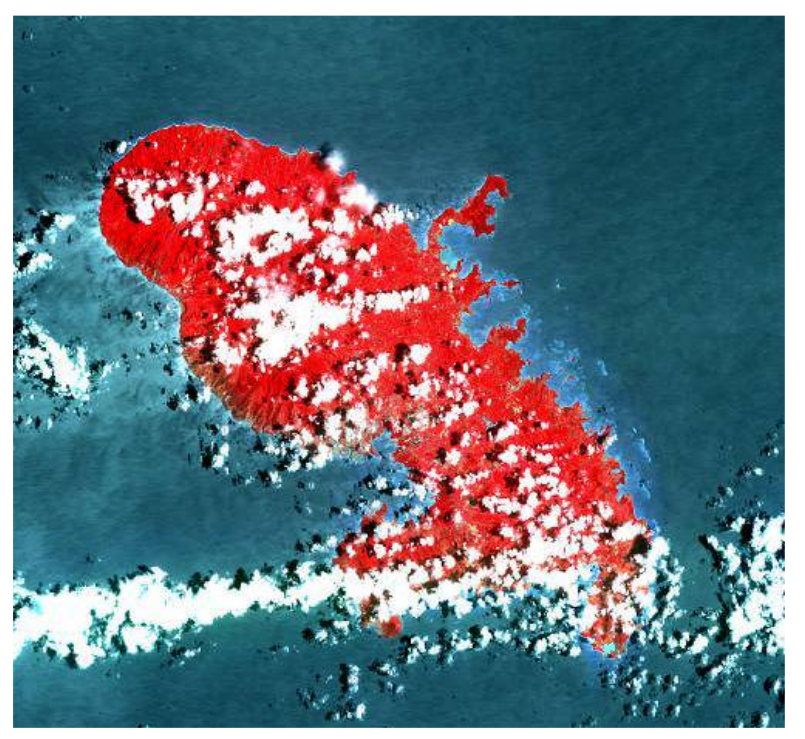

\section{Martinique}

Soil erosion risk assessment on the "Baie du Robert" watershed 


\section{$\rightarrow$ Geo-indicators to answer users needs}

- Synthetic vision of a problem in order to better understand it

- Based on reliable and easily accessible data

- Sensitive to expected changes

- Understood and accepted by its users

- Meet spatial criteria (calculated from spatial data)

- Answer agri-environnemental issues

$\Rightarrow$ Focus on the spatial relationships that exist between a parcel and its environment 
$\rightarrow$ Aim

- To provide non GIS specialists with a simple tool to assess agrienvironmental issues

- To ease the exchange \& share of information between users

- To implement the geo-indicators and enhance their dynamic nature

- To disseminate the map products derived from satellite image processing

$\rightarrow$ Selected technologies

- Open Source

$\square$ Database : POSTGIS

$\square$ Map Server : Geoserver

$\checkmark$ Interface : OpenLayers

- Meeting with OGC standards

$\square$ WMS, WFS, SLD \& compatibility future standards, WPS

- Customisable

$\checkmark$ Interface

$\square$ Geo-Indicators

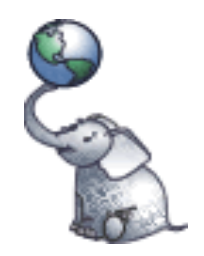

\section{Geoserver}

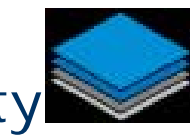




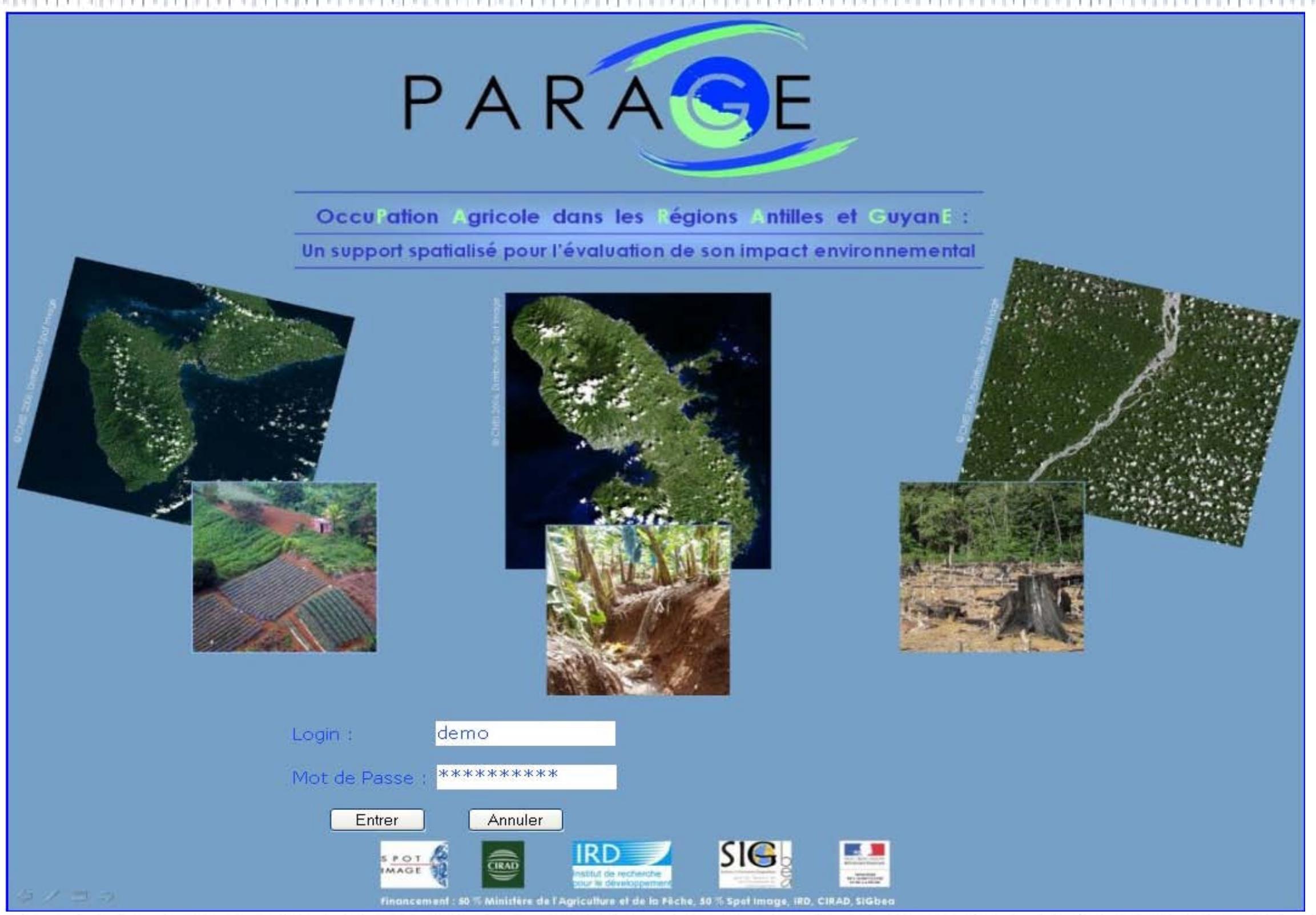


$\rightarrow$ Issue \& Study site:

- Soil erosion risk assessment on the "Baie du Robert" watershed

2 Geo-indicator: "Soil sensitivity to erosion "

- Adapting the PRAM-Cemagref soil erosion risk assessment model

4 layers of information:

$\rightarrow$ Land cover

$\rightarrow$ Slope

$\rightarrow$ Run-off accumulation

$\rightarrow$ Soil condition

$\square$ Calculation of a sensitivity index according to a decision-tree

$\rightarrow$ Map products:

- Land cover map

- Slope map

- Slope length map

- Soil map

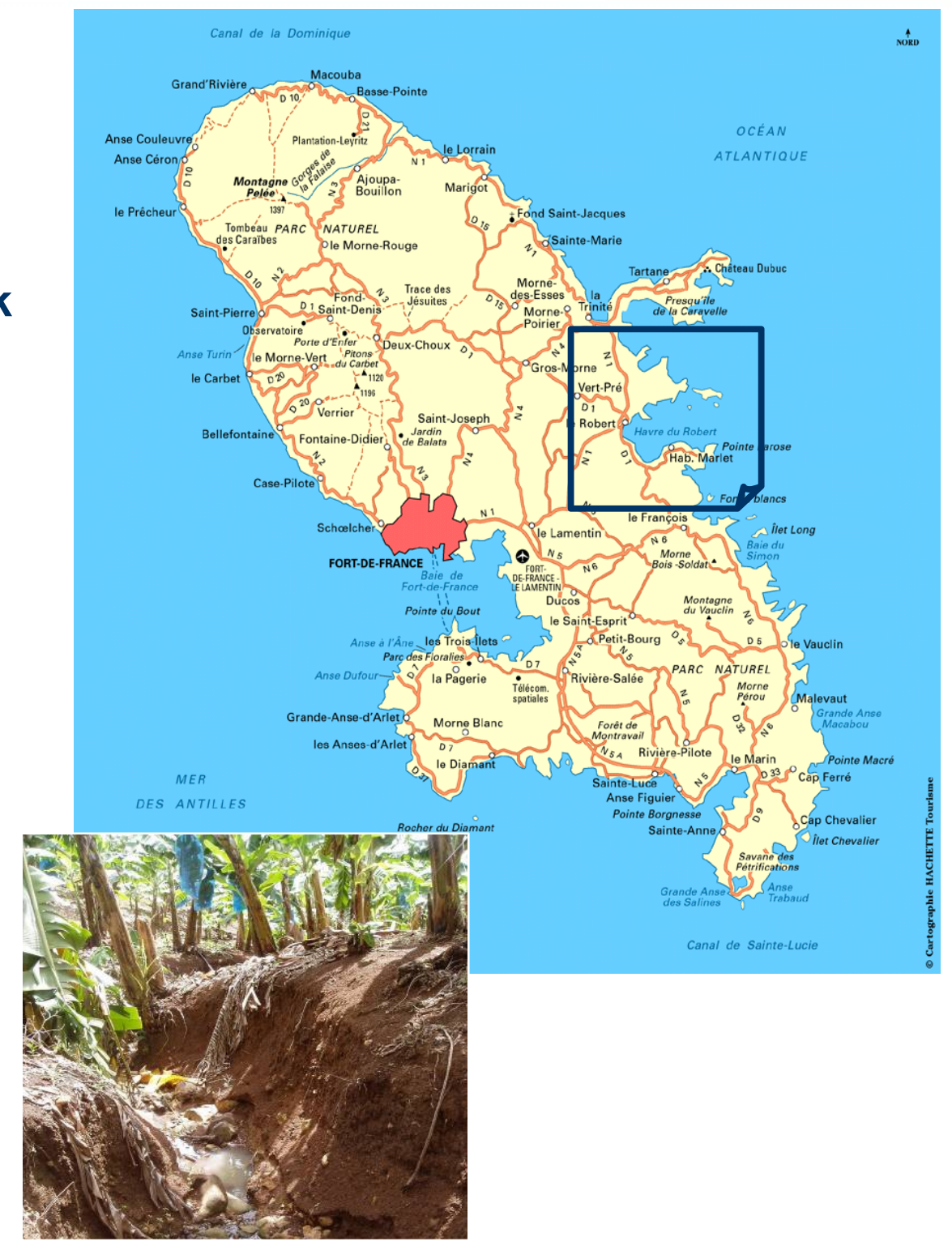




\section{Martinique: "La Baie du Robert"}

PARAGE

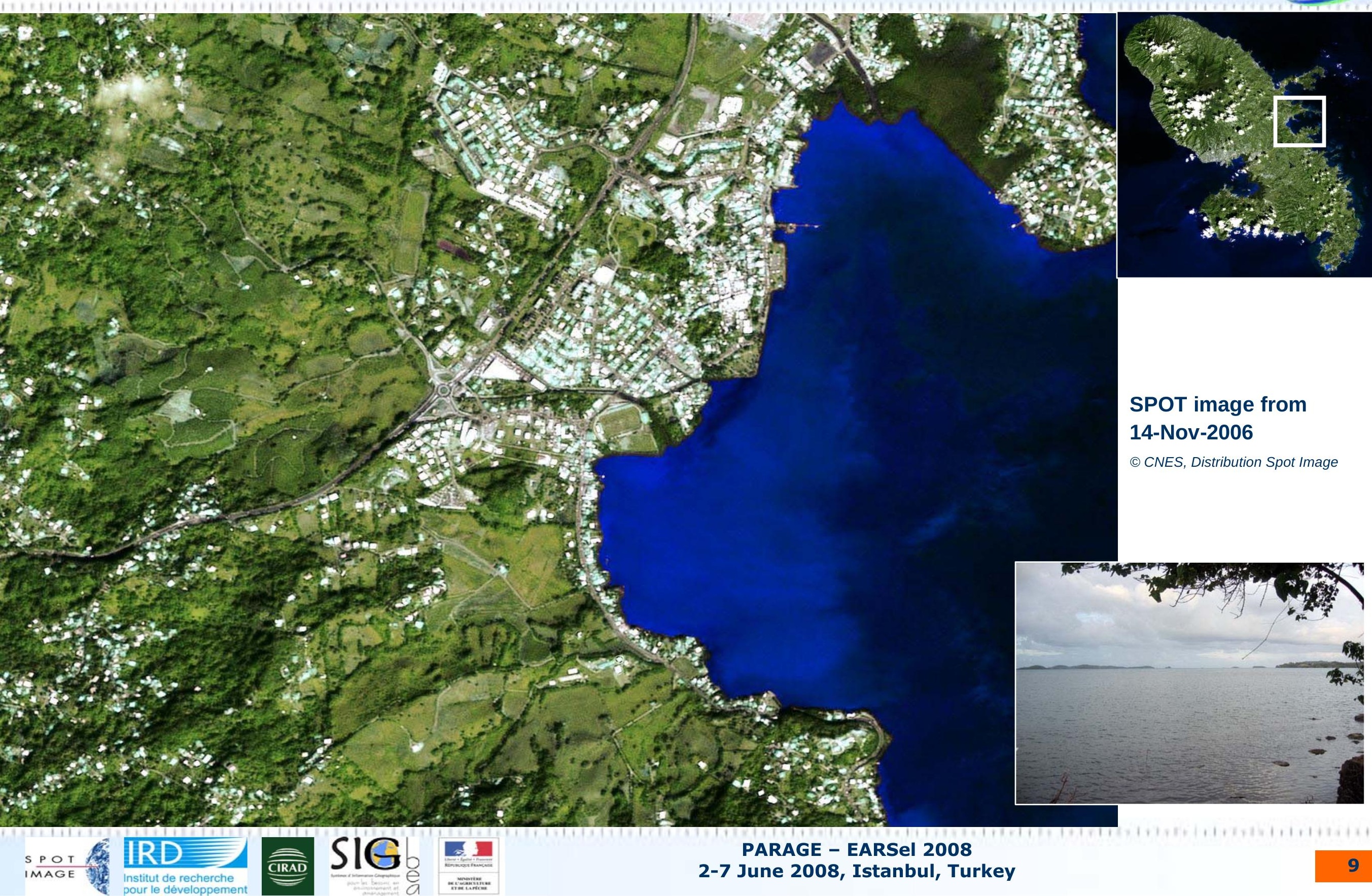




\section{$\Rightarrow$ Satellite image classification (14-Nov-2006) simplified}

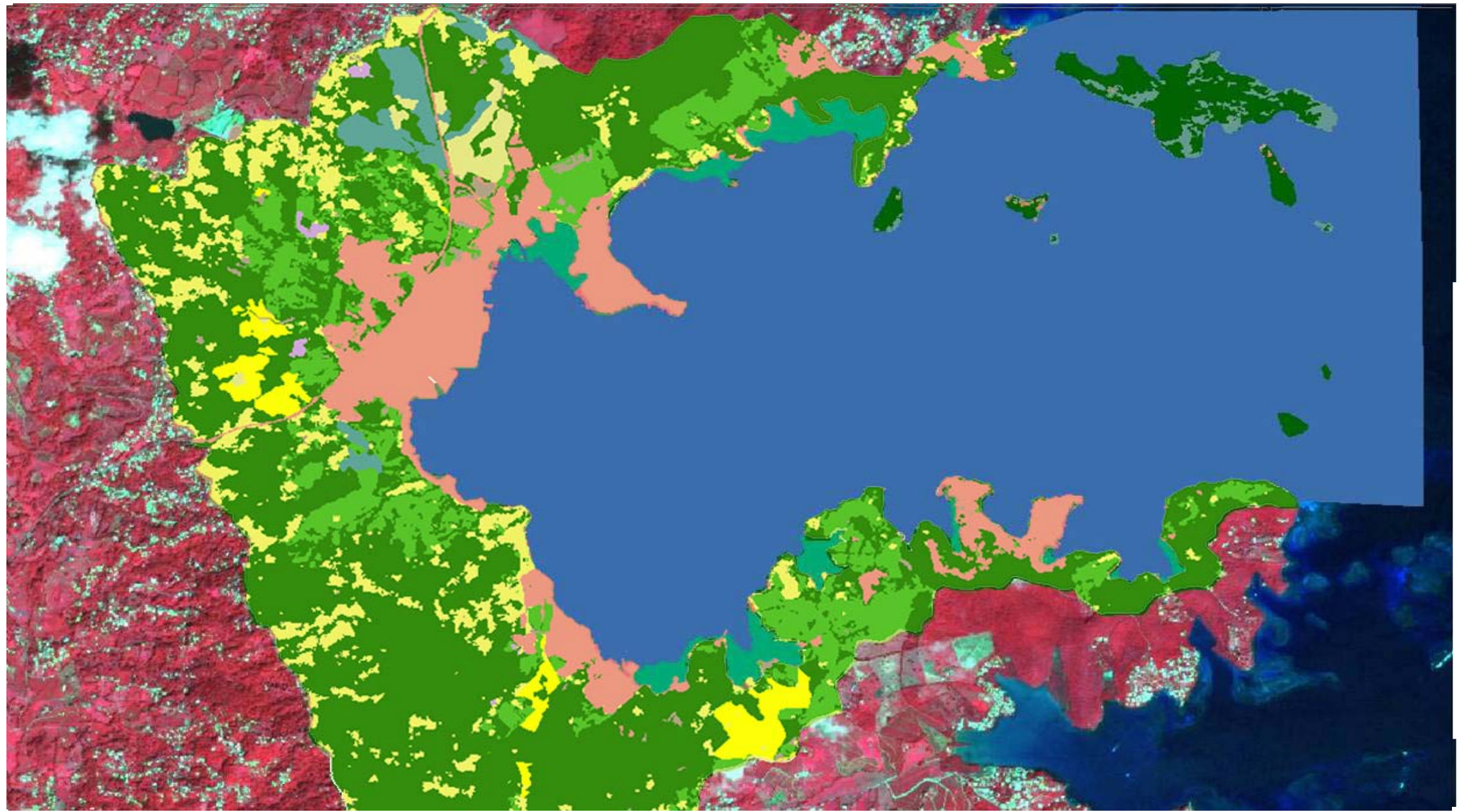

$\square$ Urbain

$\square$ Woodland

$\square$ Grassland

$\square$ Mature sugarcane

$\square$ Harvested sugarcane

$\square$ Discontinuous urban fabric

$\square$ Banana plantation $>2$ years

$\square$ Banana plantation $<2$ years

$\square$ Market gardening

$\square$ Bare soil 
기 Démonstrateur Parage - Mozilla Firefox

Eichier Édition Affichage Historique Marque-pages Qutils ?

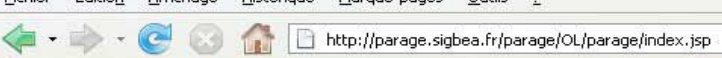

$\rightarrow$ G. $\cdot$ Goog

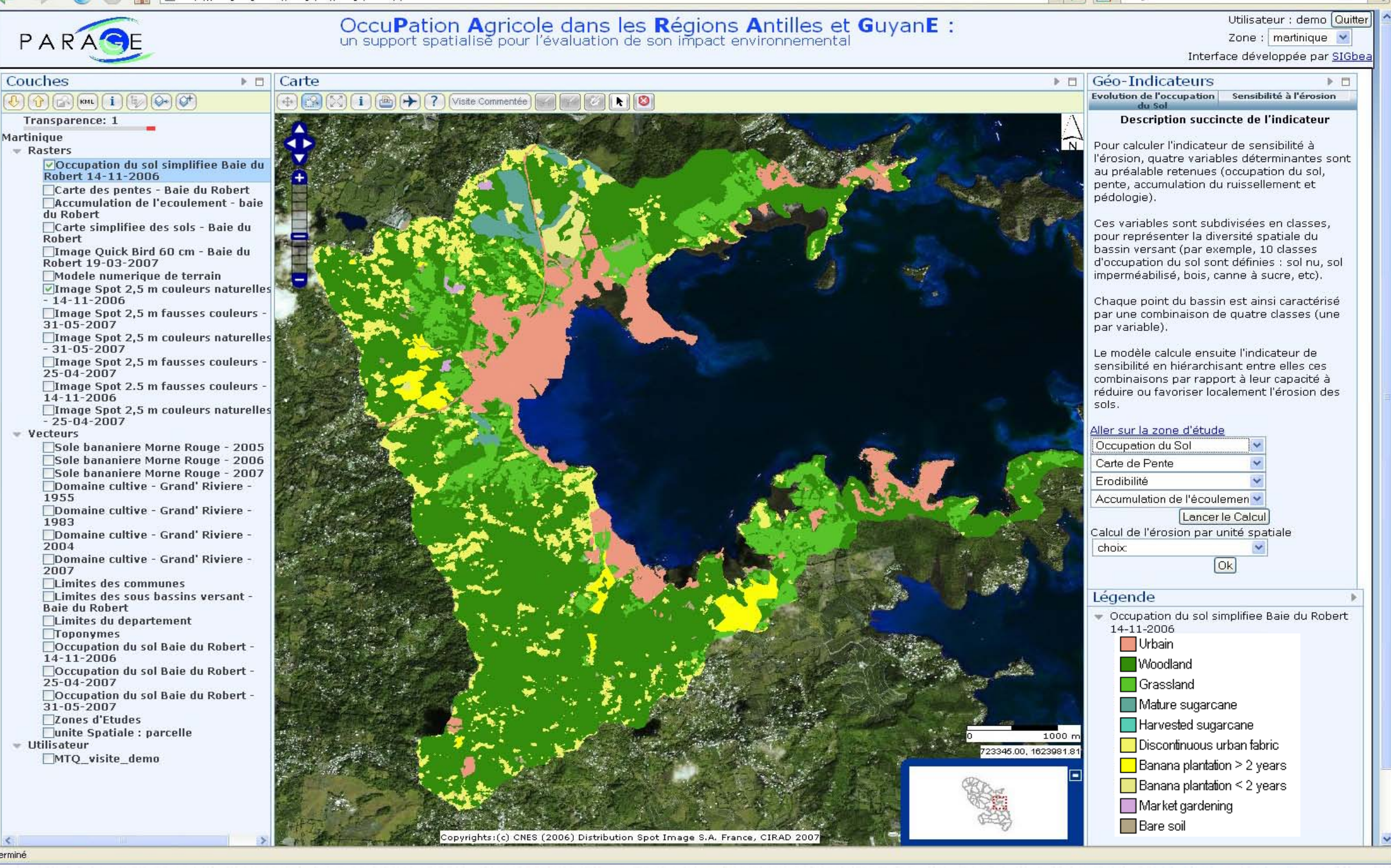




\section{Martinique: Geolnd. calculation in the PARAGE GIS Prototype}

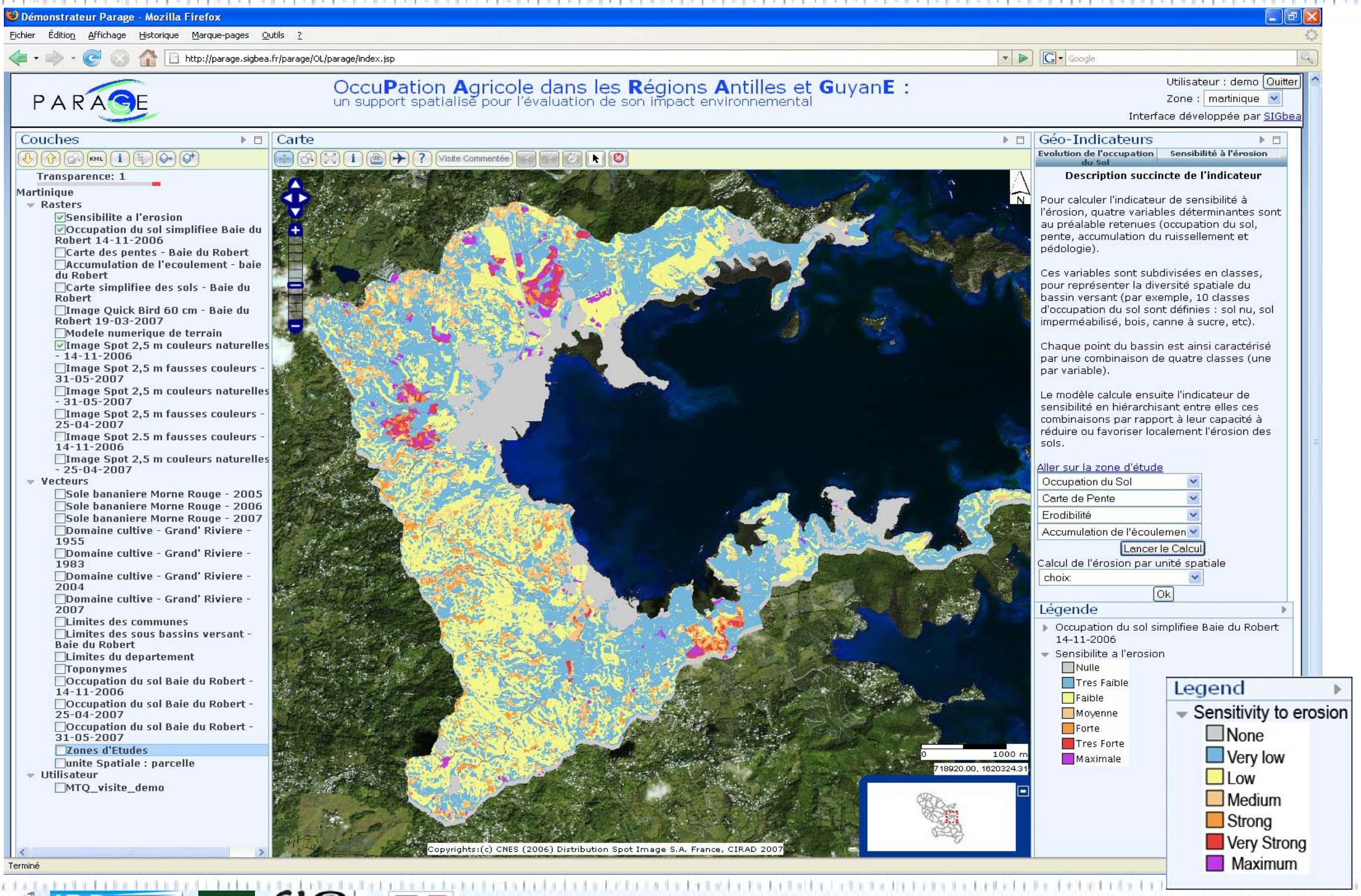



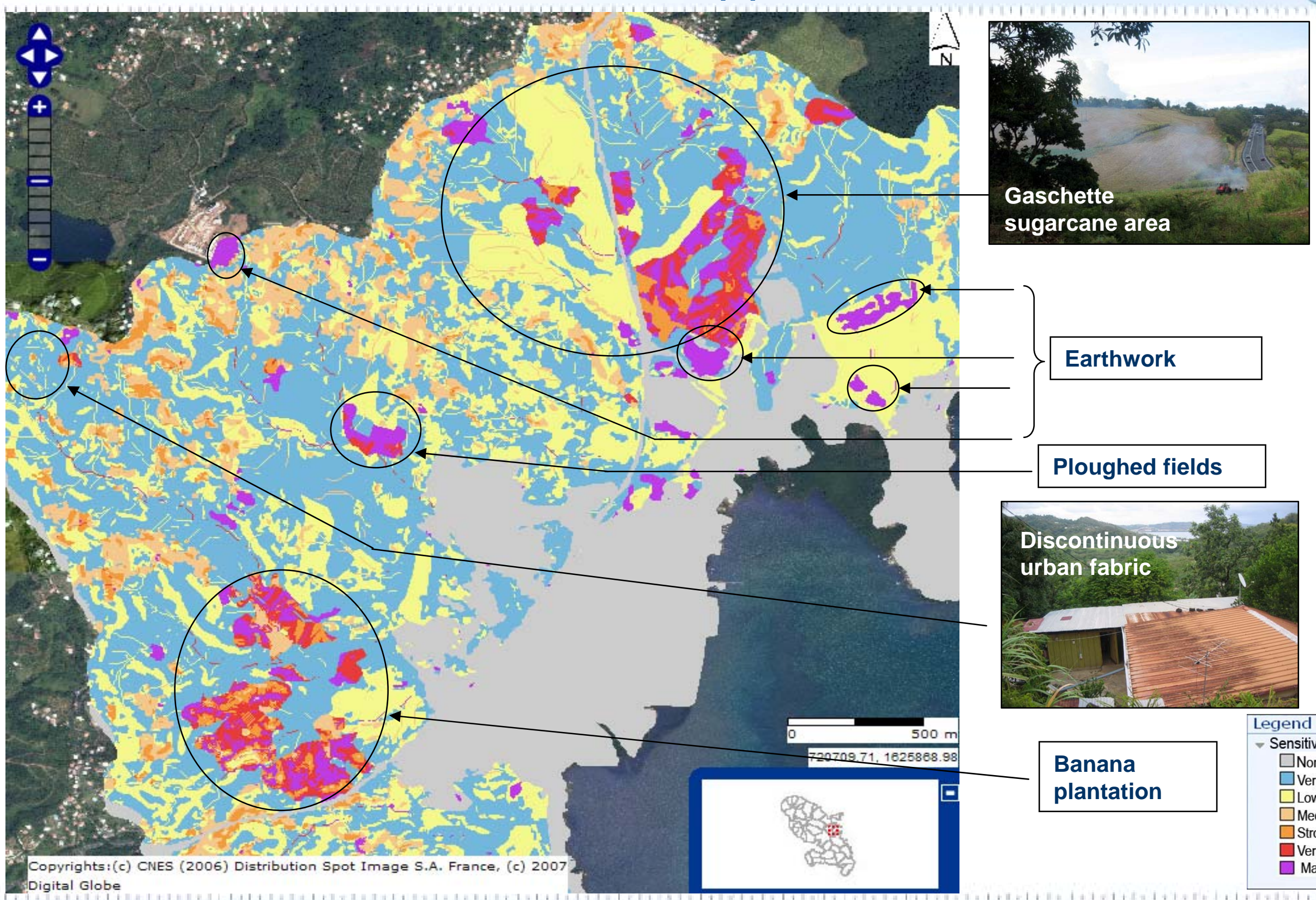

sugarcane area 
$\rightarrow$ Issue \& Study site:

- Evaluation of the agricultural pressure over sensitive or protected environments

$\square$ Grand Cul-de-Sac Marin nature reserve (coastal wetland vegetation)

o Geo-indicator: "Landscape evolution "

- assessment of changes in land cover between 2 or more dates

$\rightarrow$ Map products:

- Land cover map

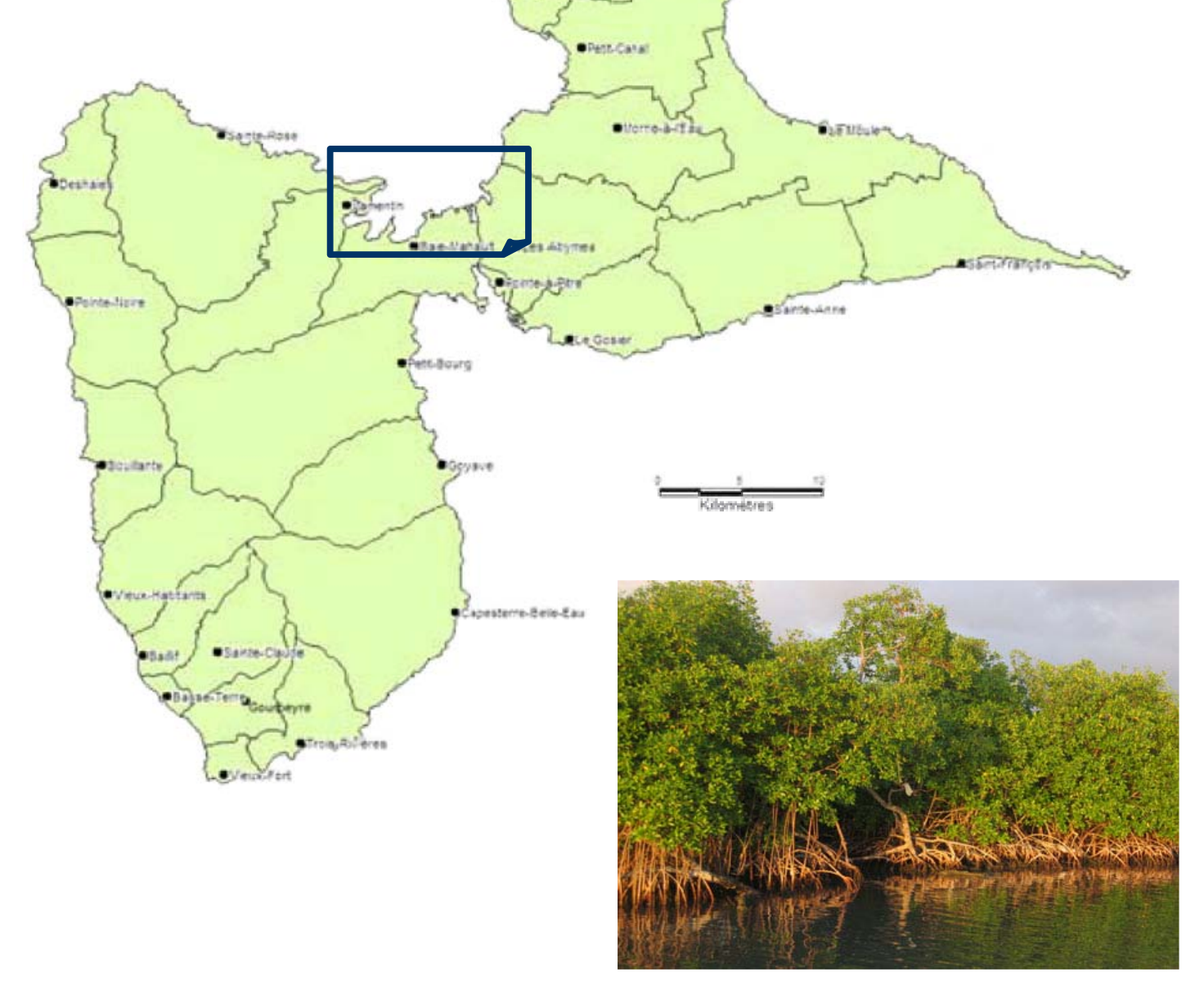


$\rightarrow$ History map nomenclature harmonisation

- Wetlands DGRST 1989

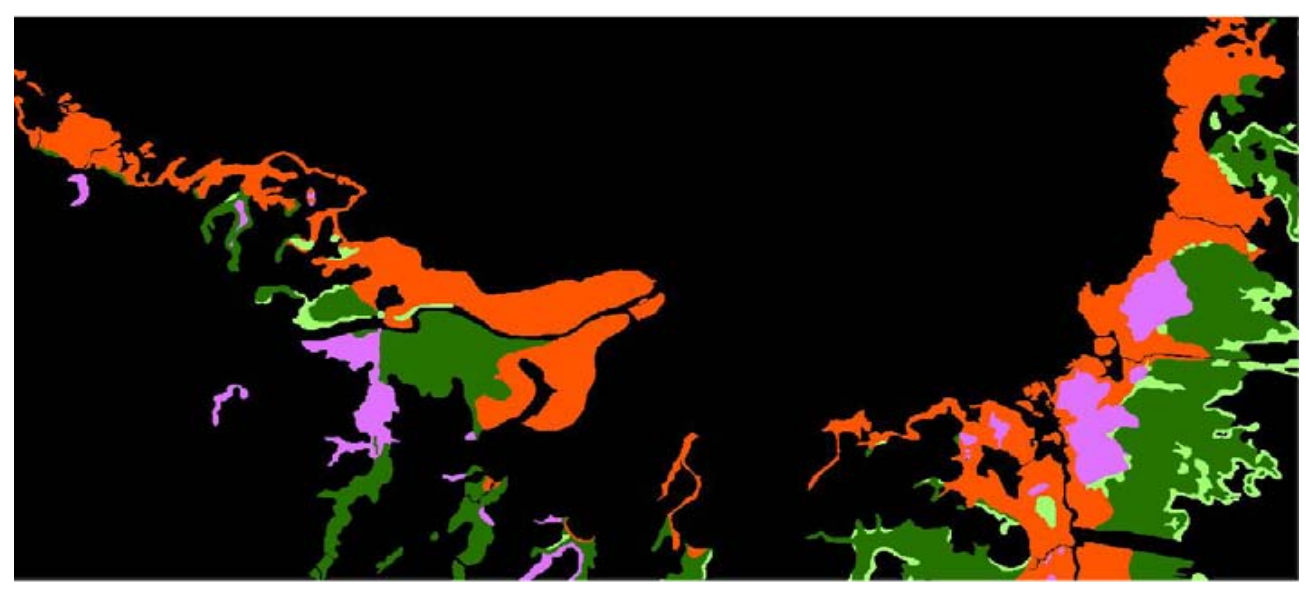

0, Unclassified

1, Mangrove forest

2, Marsh

3, Swamp forest

4, Grassland

- Wetlands ONF 1995

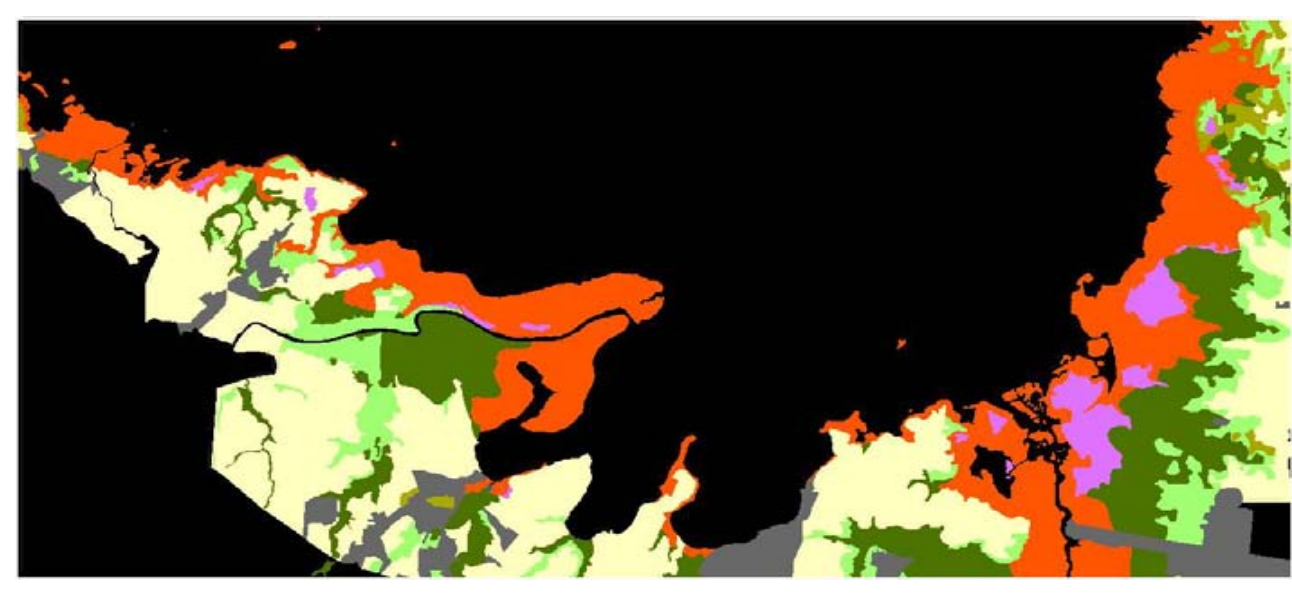

0, Unclassified

1, Mangrove forest

2, Marsh

3, Swamp forest

5 , Clouds

6, Cropland

7 , Water

8, Urban

9, Other natural formation

=> Integrated in the GIS prototype for Geo-Indicator calculation... 
$\rightarrow$ SPOT satellite image classification (20-Dec-2006) harmonised nomenclature

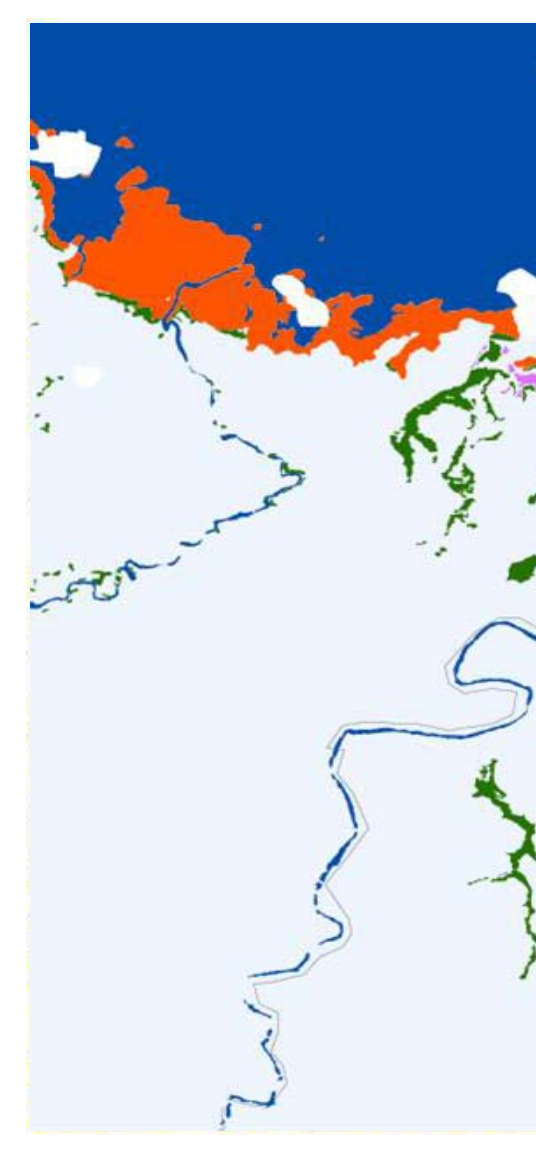

Processing CIRAD
Legend

Mangrove

Marsh

Water

Other

Clouds
Swamp forest
0 , Unclassified

1, Mangrove forest

2, Marsh

3, Swamp forest

5 , Clouds

6, Cropland

7, Water

8, Urban

9, Other natural formation 
33 Démonstrateur Parage - Mozilla Firefox

Eichier Édition Affichage Historique Marque-pages Qutils ?

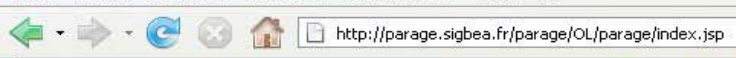

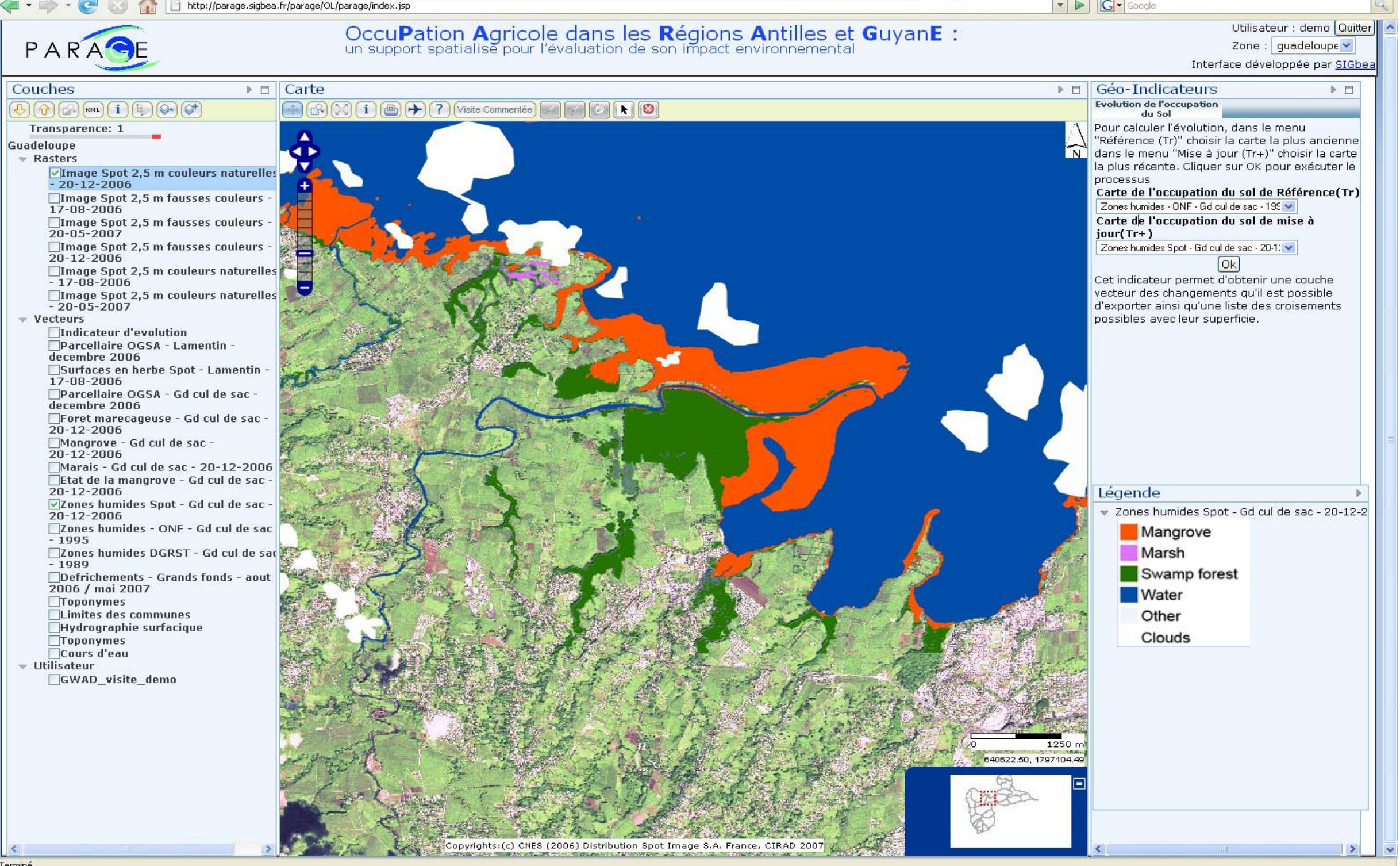
tement 


\section{Guadeloupe: Geolnd calculation in the PARAGE GIS Prototype}

Eichier Édition Affichage Historique Marque-pages Qutils

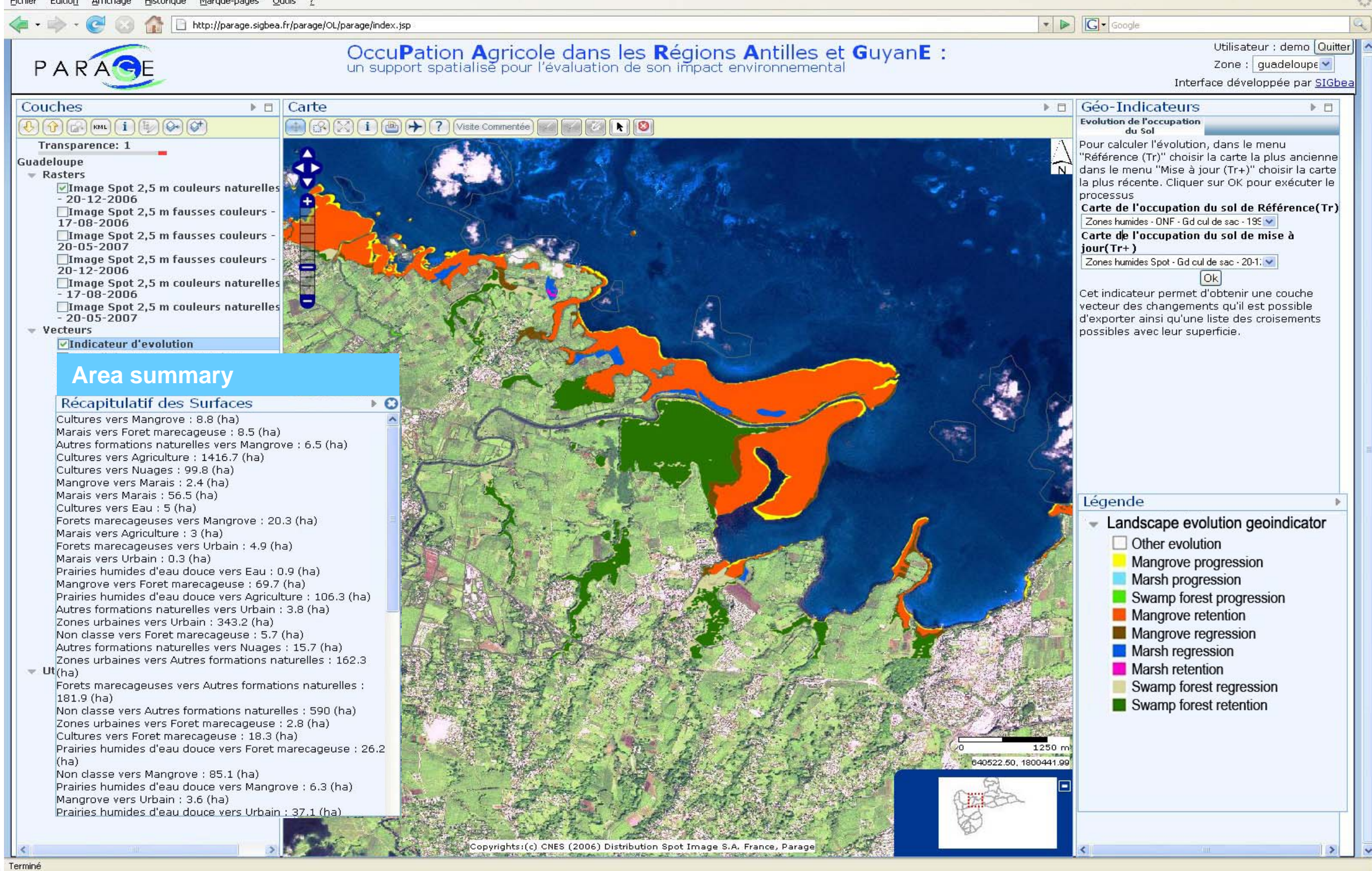


$\rightarrow$ Issue \& Study Site:

- Deforestation monitoring

- Traditional slash-and-burn agriculture

$\checkmark$ Uncontrolled clearance

$\checkmark$ UAA tripled in 20 years

- Saint-Laurent / Apatou road under construction

o Geo-indicator: "Landscape evolution"

- assessment of changes in land cover/use between 2 or more dates

$\rightarrow$ Map products:

- Land cover map

- Land use map

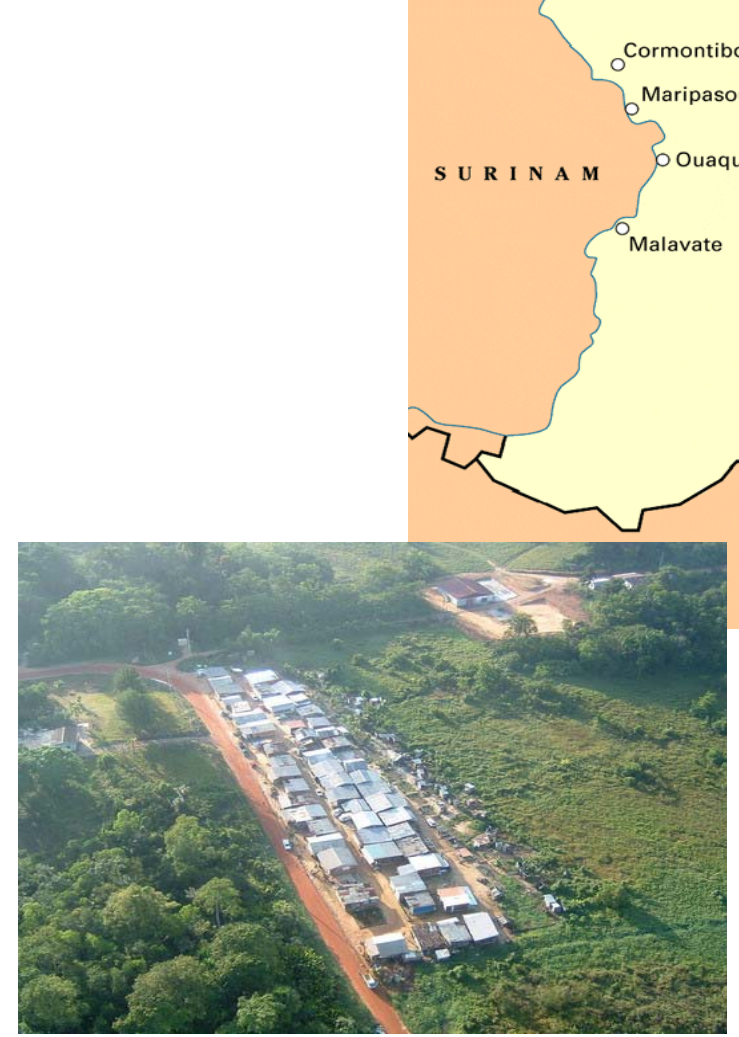

www.guyanecho.com, auteur : M. MORACCHINI PARAGE - EARSel 2008 2-7 June 2008, Istanbul, Turkey 


\section{French Guiana: clearing along the axis St Laurent - Apatou}

PAR A $\overline{G E}$

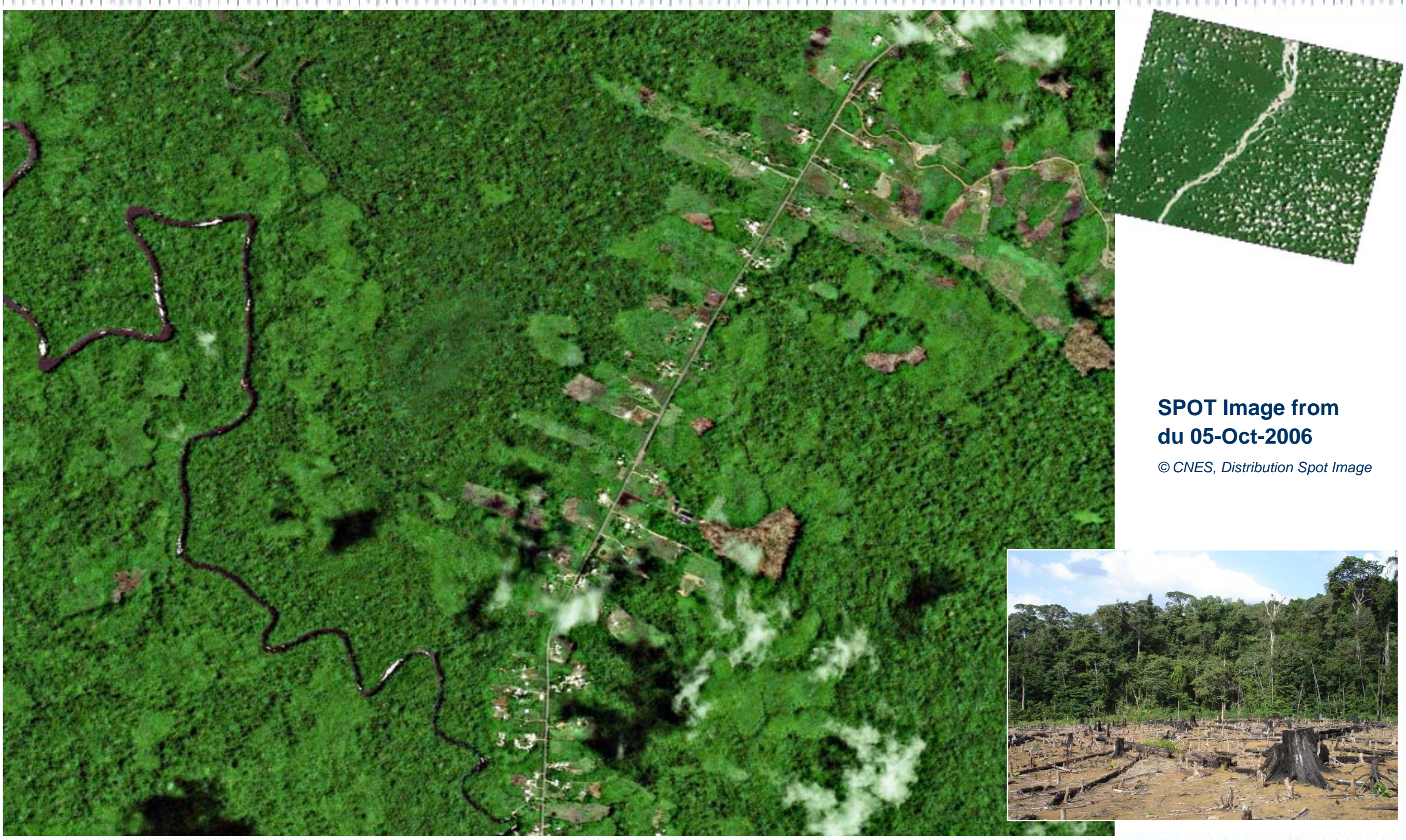




\section{French Guiana: map products}

$\Rightarrow$ SPOT satellite image classification

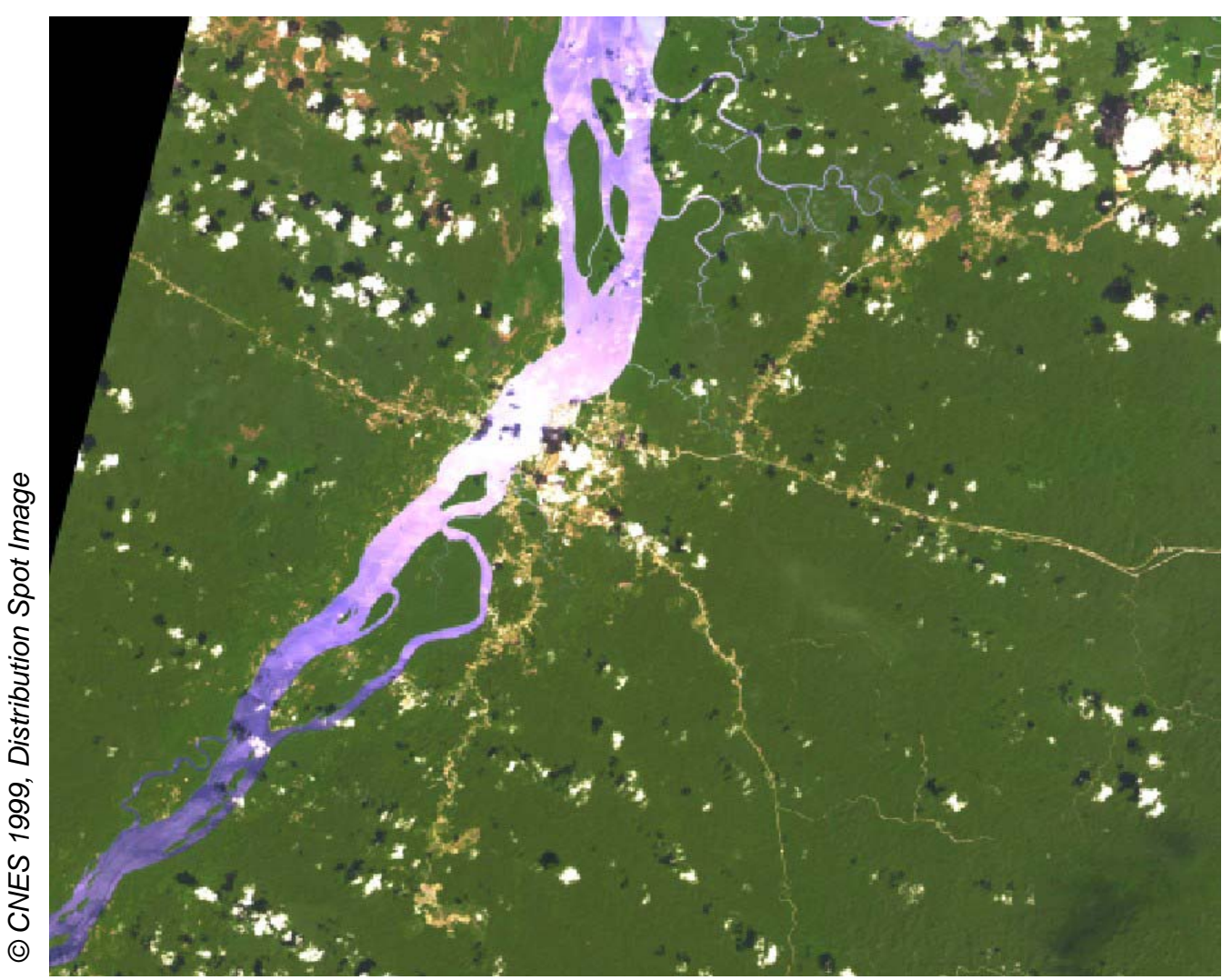

SPOT - November 26th, 1999

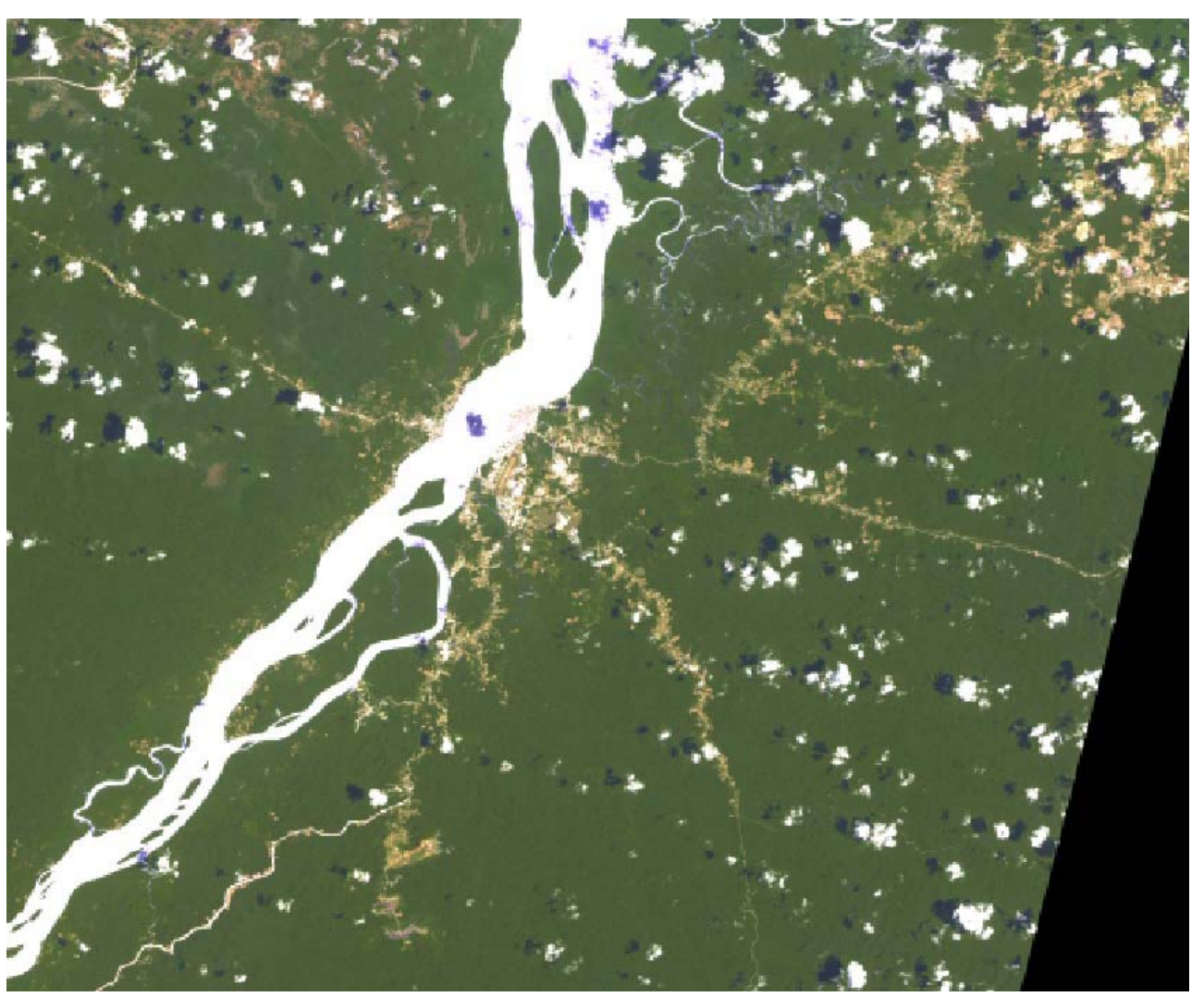

SPOT - September 26th, 2006 


\section{French Guiana: map products}

$\rightarrow$ SPOT satellite image classification

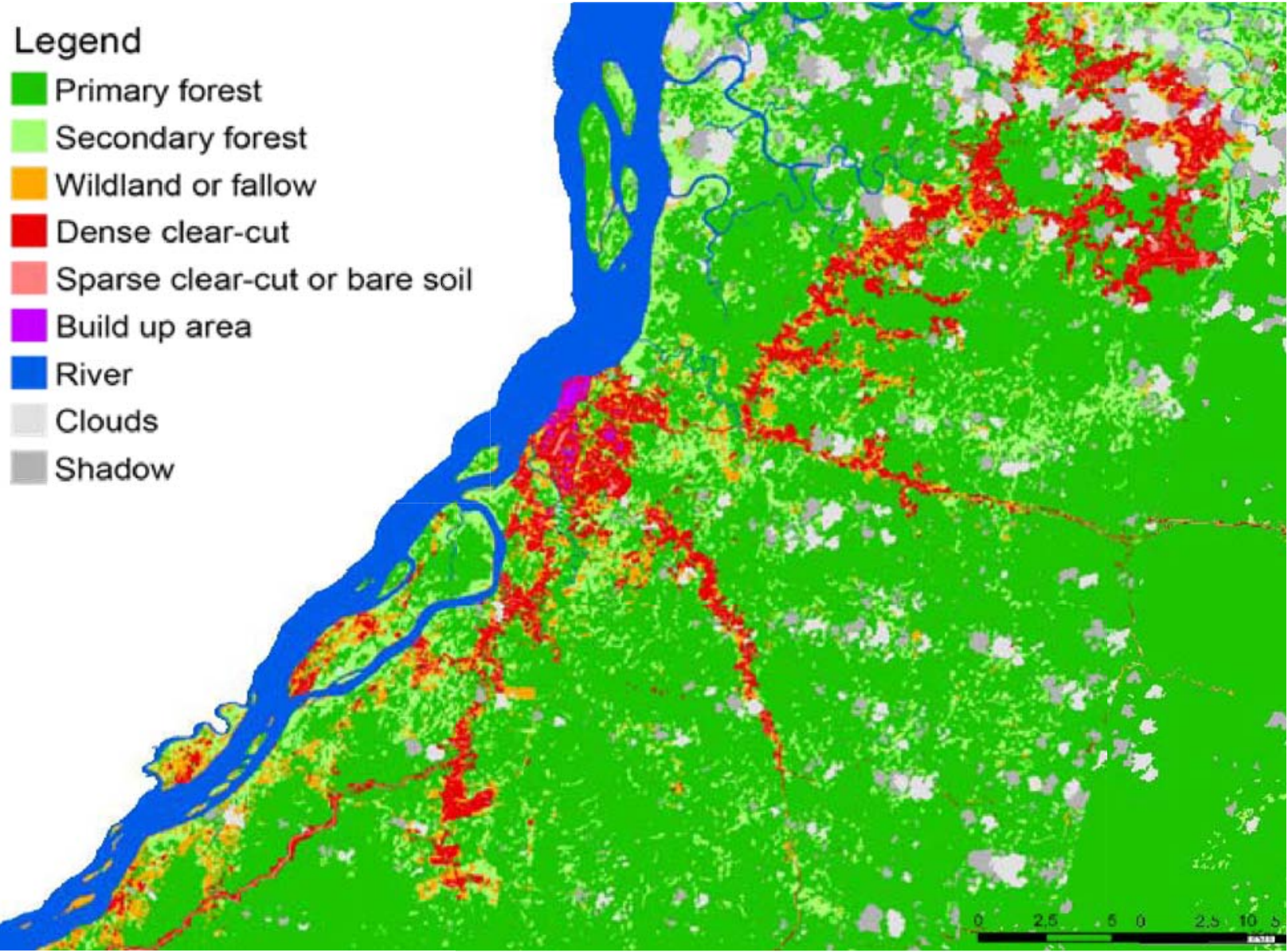

Processing IRD

Land cover map 2006

=> Integrated in the GIS prototype for Geo-Indicator calculation... 
23) Démonstrateur Parage - Mozilla Firefox

Eichier Édition Affichage Historique Marque-pages Quutils ?

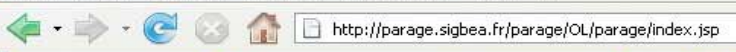

PARAGE

OccuPation Agricole dans les Régions Antilles et GuyanE :

$\rightarrow \mathrm{G} \cdot$

Utilisateur : demo [Quitter $]$ | zone: guyane

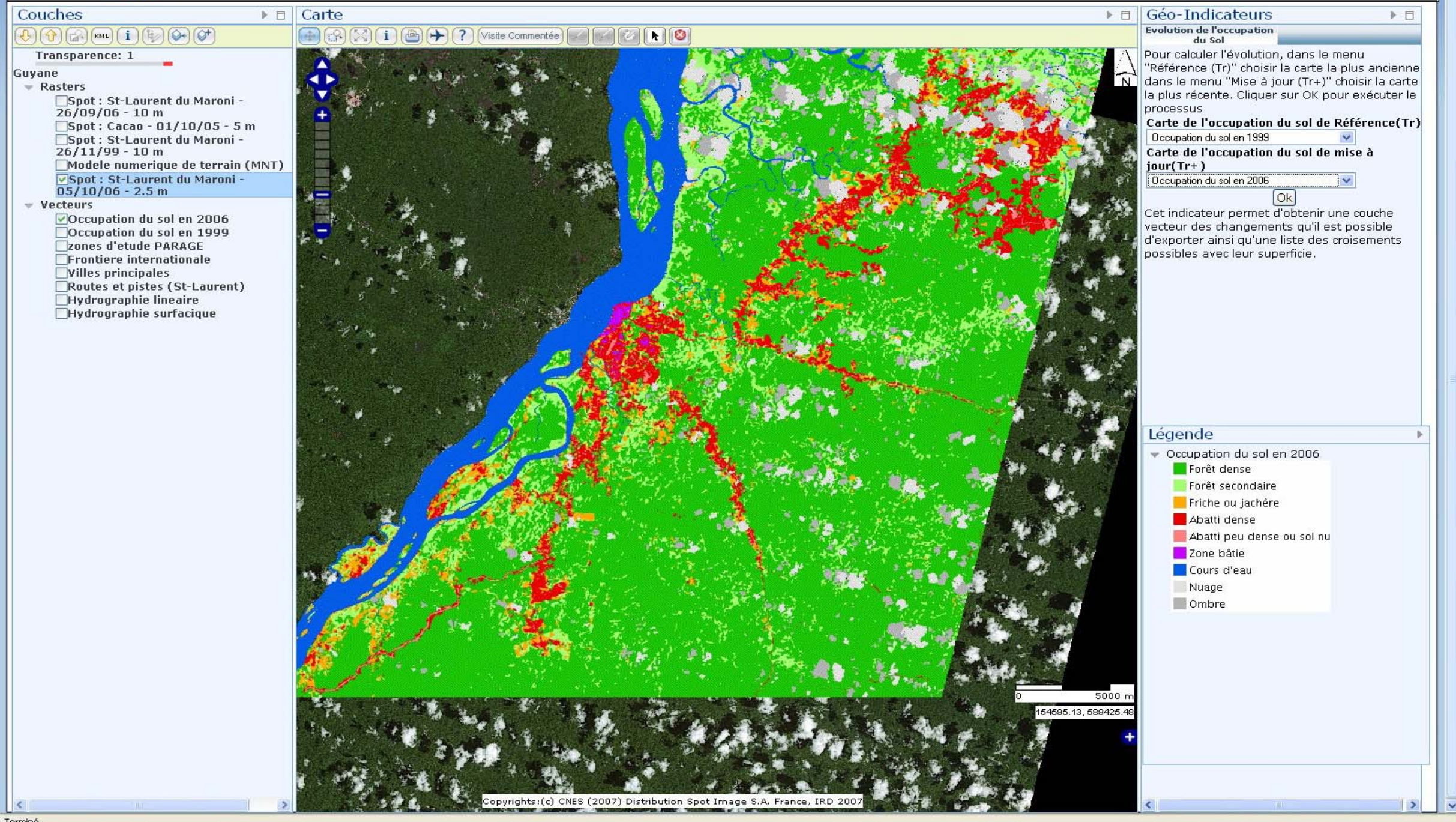




\section{French Guiana: Geolnd calculation in the PARAGE GIS Prototype}

3) Démonstrateur Parage - Mozilla Firefox

Fichier Édition Afffichage Historique Marque-pages Qutils

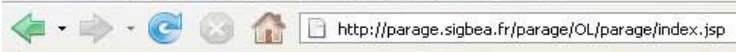

$$
\begin{array}{ll}
\text { PARAGE } & \text { OccuPation Agricole dans les Régions Antilles et GuyanE : } \\
\text { un support spatialise pour l'évaluation de son impact environnemental }
\end{array}
$$

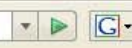

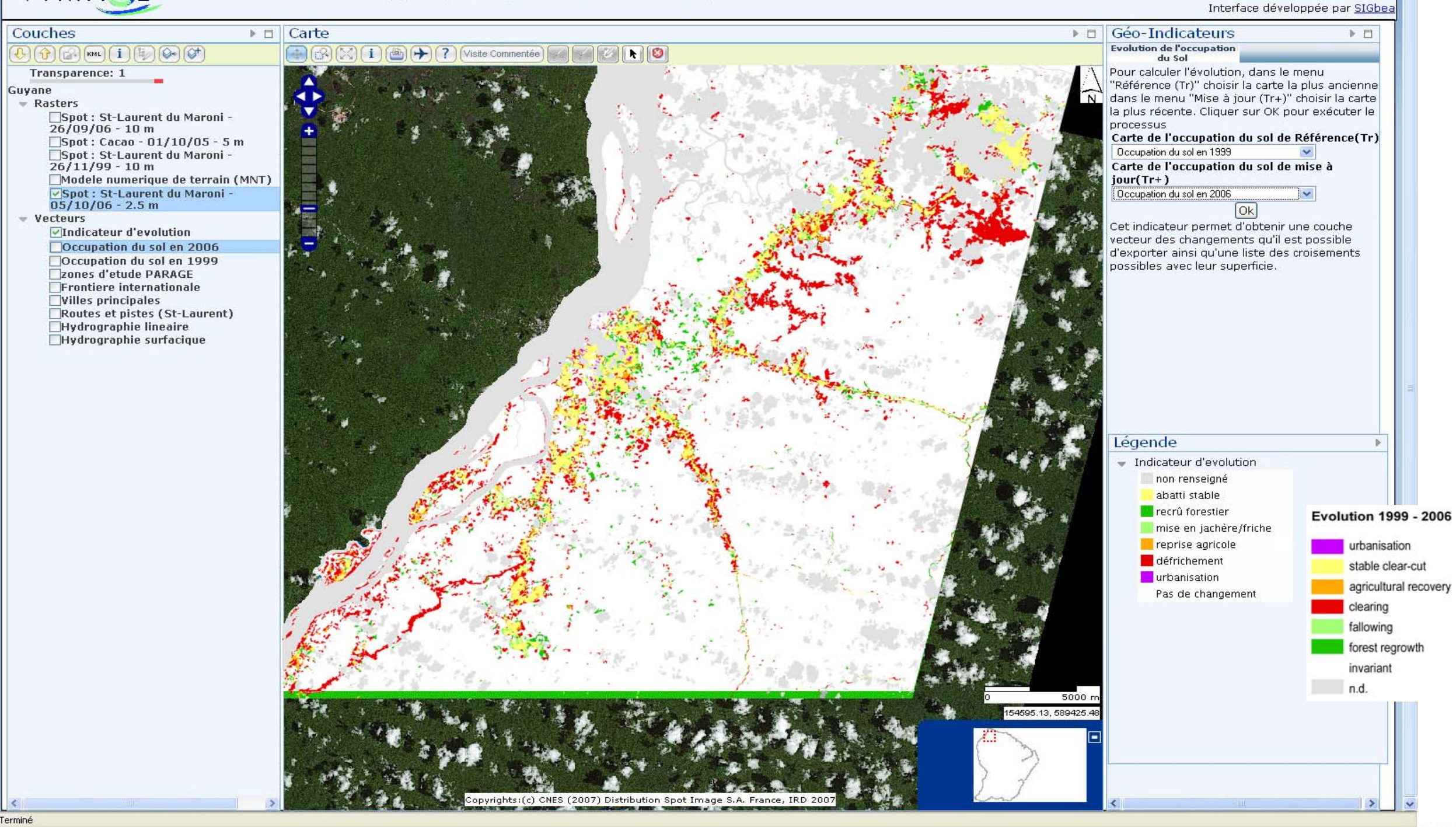




\section{Conclusion and Perspective}

$\rightarrow$ Conclusion (user evaluation)

- Map products and Geo-indicators

$\square$ useful information for territory knowledge diagnosis and monitoring

$\square$ map products and Geo-indicators can be updated regularly (satellite based)

$\square$ methodology applicable in other territories

- GIS prototype

$\square$ Web \& open source $=>$ no need for install

$\square$ from GIS experts to general public

a simple communication tool

$\square$ information sharing and dissemination

- Limits

$\square$ available internet bit rate

$\square$ climatic conditions

$\square$ market gardening or crops under shade issues

$\Rightarrow$ Towards an operational service

- Geographic extension

- Product standardisation

- Tool robustness
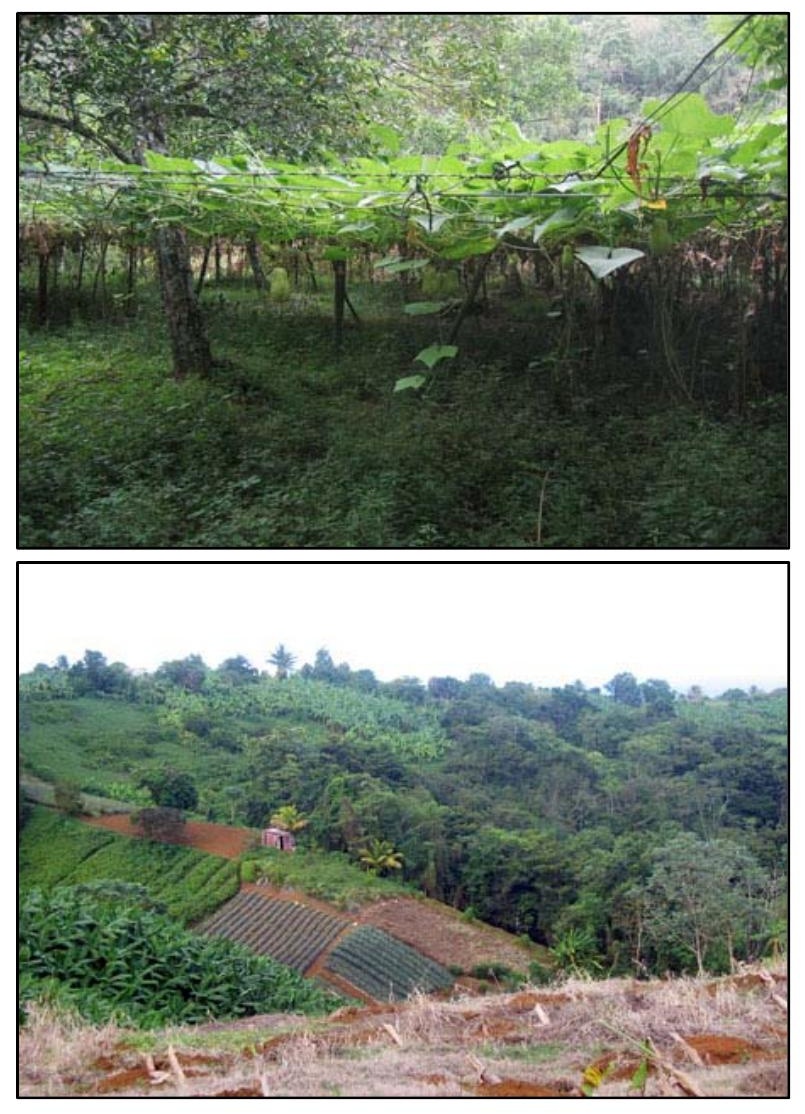
Jean-Marie FOTSING fotsing@orleans.ird.fr

\section{Florence BAILLARIN}

Florence.baillarin@spotimage.fr

\section{IRD}

Institut de recherche pour le développement
Site Web
http://www.spotimage. $\mathrm{frl}$
\& contacts :
Projet PARAGE

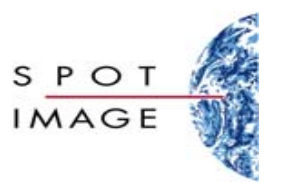

Gérard LAINE

gerard.laine@cirad.fr

Béatrice ARIAUX

bariaux@sigbea.fr 